\title{
Entwicklung und Erprobung eines hochsensitiven und miniaturisierten Wasserstoffmesssystems
}

\author{
Pramit Sood ${ }^{1,2}$, Jens Zosel', Michael Mertig ${ }^{1,2}$, Wolfram Oelßner ${ }^{1}$, Olaf Herrmann ${ }^{3}$, Michael Woratz ${ }^{3}$ \\ ${ }^{1}$ Kurt-Schwabe-Institut für Mess- und Sensortechnik e.V. Meinsberg (KSI), Waldheim \\ ${ }^{2}$ Technische Universität Dresden, Professur für Physikalische Chemie, Mess- und Sensortechnik, \\ Dresden \\ ${ }^{3} \mathrm{ACl}$ Analytical Control Instruments $\mathrm{GmbH}$, Berlin \\ Kontakt: pramit.sood@ksi-meinsberg.de
}

\section{Einleitung}

Zwei der größten Herausforderungen für die Zukunft der Energiewirtschaft sind deren Erneuerbarkeit und Nachhaltigkeit. Alle modernen Einschätzungen der globalen Energiezukunft gehen davon aus, dass der Zunahme des Energiebedarfs und der Minderung der Treibhausgasemissionen zunehmend durch einen vielfältigen Energiemix begegnet werden muss, der gleichzeitig umweltfreundlich ist [1]. Wasserstoff $\left(\mathrm{H}_{2}\right)$, der bereits umfassend in vielen Industriezweigen für eine Vielzahl von Anwendungen [2] genutzt wird, bietet als Energieträger ein großes Potenzial, um den oben genannten Herausforderungen der Emissionsreduzierung, Erneuerbarkeit und Diversifizierung zu begegnen. [3]. Dazu ist einerseits der Einsatz von $\mathrm{H}_{2}$ zu verbreitern, was beispielsweise in Anwendungen wie Brennstoffzellenfahrzeugen [4] angestrebt wird, und andererseits seine Produktion aus erneuerbaren Quellen wie Wind-, Wasser- und Solarkraftwerken zu intensivieren (power-to-gas) [5].

Eine Dezentralisierung der $\mathrm{H}_{2}$-basierten Energieversorgungskette wird somit den Consumerbereich in weitaus größerem Umfang betreffen, als dass heute der Fall ist und neben technischen und wirtschaftlichen Aspekten, neue Herausforderungen bei der Anwendungssicherheit mit sich bringen. Diese müssen unbedingt adressiert werden, um die Akzeptanz dieses Energieträgers in der öffentlichen Nutzung nicht zu gefährden [6]. Da $\mathrm{H}_{2}$ ein sehr leichtes, leicht entzündliches und geruchloses Gas mit einem breiten Explosionsbereich an Luft darstellt, besteht ein allseits akzeptierter Ansatz für das Risikomanagement in seiner automatisierten hochselektiven Onlinemessung in einem breiten Konzentrationsbereich bis unter $<10 \mathrm{Vol}$.ppm, so dass eventuelle Leckagen frühzeitig detektiert werden können [7].

Dieser Ansatz erfordert eine komplexe Infrastruktur zur Sicherheitsüberwachung, die auf hochsensitiven, selektiven und langzeitstabilen Sensoren und Messsystemen besteht, die in flexiblen Feldinstallationen eingesetzt werden können. In [8] werden dazu verschiedene kommerzielle Sensortechnologien und Prüfverfahren zur $\mathrm{H}_{2-}$ Leckerkennung und Sicherheitsüberwachung beschrieben und bewertet. In [9-10] wird die Verwendung eines coulometrischen Festelektrolyt-Gassensors (FES) als GCDetektor beschrieben, der Spurenkonzentrationen von oxidierbaren Gaskomponenten $\left(\mathrm{H}_{2}, \mathrm{CH}_{4}\right)$ in sauerstoffhaltigen Gasgemischen erfasst. Als Festelektrolyt kommt Zir- coniumdioxid zum Einsatz, das mit Yttriumoxid stabilisiert wurde (YSZ). Der Sensor misst bei $600-800{ }^{\circ} \mathrm{C}$ einen Elektrolysestrom, der sich bei der Oxidation oder Reduktion von chromatographisch getrennten Gaskomponenten ergibt. Dieser Strom entsteht, indem Oxidionen an der Dreiphasengrenze (Pt/YSZ/Gasphase) der Arbeitselektrode aus einem YSZ-Sauerstoffionenleiter in die Reaktionszone übertreten. Die Arbeitselektrode ist gegenüber einer Pt-Referenzelektrode polarisiert, um einen konstant niedrigen Sauerstoffpartialdruck im Trägergas zu erzeugen [10]. GI. 1 beschreibt die Beziehung zwischen der Konzentration $(\varphi)$ des Analyten ( $\mathrm{x}$ ) und dem Detektorsignal (I) basierend auf dem Faraday'schen Gesetz:

$$
\boldsymbol{\varphi}(\mathbf{x})=\frac{\mathbf{Q} \cdot V_{\mathbf{m}}}{\mathbf{z} \cdot \mathbf{F} \cdot V_{\mathbf{s}}}=\frac{V_{\mathbf{m}}}{\mathbf{z} \cdot \mathbf{F} \cdot V_{\mathbf{s}}} \int_{\mathbf{t}_{\mathbf{s}}}^{\mathbf{t}_{\mathrm{e}}}\left(\mathbf{I}-\mathbf{I}_{\mathbf{B}}\right) \mathbf{d t}
$$

wobei $t_{s}$ und $t_{e}$ die Start- und Endzeiten des Peaks sind. $F$ bezeichnet die Faraday-Konstante, $I_{B}$ steht für den Basislinienstrom und $z$ für die Anzahl der in der Reaktion übertragenen Elektronen $\left(z=4\right.$ bei $\left.\mathrm{O}_{2}\right) . V_{m}$ und $V_{s}$ stehen für das Molvolumen des Gases bzw. für das eingespritzte Probevolumen. Der Detektor wurde in Bezug auf Materialeigenschaften und instrumentelle Rauschquellen charakterisiert und optimiert, um eine selektive Messung von $\mathrm{H}_{2}$ Konzentrationen bis hinunter zu 500 Vol.-ppb [10] zu ermöglichen. Im vorliegenden Beitrag werden Arbeiten in zwei thematische Richtungen dargestellt, die einerseits die Miniaturisierung und Charakterisierung der wesentlichen Bauteile des Messsystems und andererseits die umfassende Charakterisierung der wesentlichen Sensorparameter unter Einfluss der verschiedenen Mess- und Betriebsbedingungen betreffen.

\section{Methoden und Materialien}

Zur Charakterisierung von Sensorparametern und Messbedingungen wurde der in Abb.1 gezeigte GasChromatograph (C) (8610C, MG \#1, SRI Instruments Europe $\mathrm{GmbH}$, Bad Honnef) mit einer kommerziell verfügbaren Sauerstoffpumpzelle (D) (O2-DF-28.0, Zirox GmbH, Greifswald) mit Temperierung (F) und einer selbst entwickelten potentiostatischen Regelschaltung (E) kombiniert [10]. Die Gasprobe wird in einem bei $60 \pm 1^{\circ} \mathrm{C}$ temperierten 10-Port-Rotationsventil (A) mit Probeschleife des Volumens $1 \mathrm{ml}$ eingespritzt. Im Säulenofen (B) sind zwei gepackte Edelstahl-Säulen zur chromatographischen Tren- 

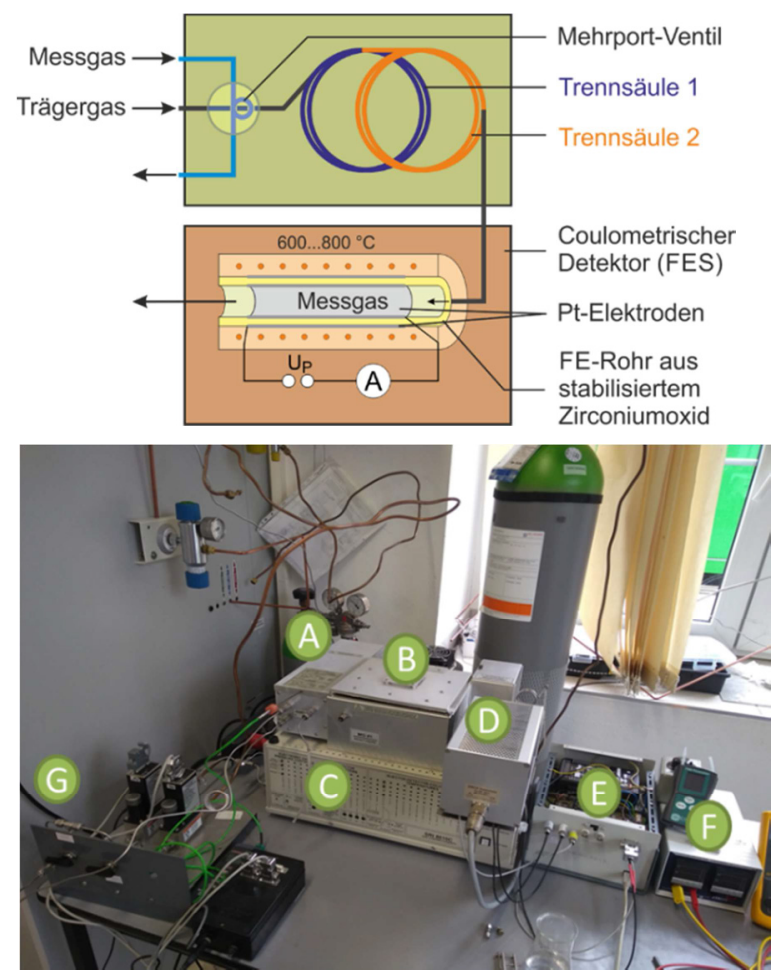

nung der Gaskomponenten $\mathrm{H}_{2}, \mathrm{CH}_{4}$ und $\mathrm{O}_{2}$ angeordnet, die ein Molekularsieb 13X/5A bzw. Silika-Gel enthalten.

Abb. 1: Schema (oben) und Bild (unten) des Versuchsaufbaus, Alphabetische Beschriftung siehe Text.

Als Trägergas wurde Stickstoff $\left(\mathrm{N}_{2}\right)$ der Qualität 5.0 verwendet. Die Probeschleife wird kontinuierlich mit Messgasgemischen gespült, die mit kalibrierten Massendurchflussreglern (G) (Brooks Instruments, Hatfield, USA) unter Verwendung von Prüfgasen mit 1000 bzw. 102,7 Vol.-ppm $\mathrm{H}_{2}$ in synthetischer Luft hergestellt wurden. Die Parameter chromatographisches Trennverhalten, Sensitivität, Genauigkeit und Reproduzierbarkeit/Langzeitstabilität wurden bei Detektortemperaturen zwischen DT $=600{ }^{\circ} \mathrm{C}$ und $750{ }^{\circ} \mathrm{C}$, Polarisationsspannungen zwischen $U_{P}=-300 \mathrm{mV}$ und $U_{P}=-450 \mathrm{mV}$, eingespritzten $\mathrm{H}_{2}$-Konzentrationen zwischen 0,2 - 180 Vol.-ppm, Trägergasvolumenströmen von 6 bis $31,5 \mathrm{ml} / \mathrm{min}$ sowie Trennsäulenlängen I = 1-2 $\mathrm{m}$ untersucht. Die Trägergasvolumenströme wurden mit einem kalibrierten Durchflussmessgerät (DryCal 800-3, Mesa Laboratories, Inc., New Jersey, USA) am Ausgang der Säulen gemessen. Bei jeder Einstellung wurden vier Chromatogramme aufgezeichnet und Mittelwerte der Peakfläche und Peakhöhe gebildet.

Neben der Charakterisierung der Sensorparameter wurden die Einspritzeinheit, der Säulenofen und der Detektor als Hauptbaugruppen eines $\mathrm{H}_{2}$-Messsystems miniaturisiert, charakterisiert und materialtechnisch optimiert. Die Einspritzeinheit besteht aus 8 einzeln angesteuerten Solenoid-Mikroventilen (S0705DX-CO, 3/2 Wege Ventile, $\mathrm{SMC} \mathrm{GmbH}$ ), die auf neu entwickelte miniaturisierte Manifolds montiert wurden, wie Abb. 2 zeigt. Diese Manifolds wurden einerseits mittels 3D-Metalldruck (Mini-Manifold 45 x $35 \times 25 \mathrm{~mm}^{3}$ ) und andererseits mittels Feinwerktechnik in Modulbauweise (Mikro-Manifold $60 \times 12 \times 10 \mathrm{~mm}^{3}$ ) angefertigt.

Die in Abb. 2 schematisch und als Bild dargestellten Manifolds wurden im Hinblick auf die Gasdichtigkeit aller Kanäle und äußeren Anschlüsse mittels Helium-Lecktest geprüft. Zur Charakterisierung des Injektionsverhaltens und der Gasausbreitung in den Manifolds wurden diese mit kurzgeschlossenen Trennsäulen-Anschlüssen an den o.g. FES-Detektor angeschlossen und mit realen Gasströmen für Mess- und Trägergas untersucht. Das Detektorsignal wurde mittels Potentiostat (Interface 1000, Gamry Instruments, Warminster, USA) über mehrere Loadund Injektionszyklen bei verschiedenen Polarisationsspannungen, Detektortemperaturen, Trägergasvolumenströmen und eingespritzten $\mathrm{H}_{2}$-Konzentrationen aufgezeichnet.

Das Designkonzept und ein entsprechendes Versuchsmuster eines miniaturisierten GC-Säulenofens wurden bereits in [11] beschrieben. Zur Charakterisierung der funktionellen Leistungsfähigkeit wurden Aufheiz- und Abkühlraten bei konstanter oder steigender Heizleistung und maximaler Kühlleistung gemessen. Der Einfluss einer Luftklappe am Luftauslass des zylindrischen Säulenträgers wurde ebenfalls getestet. Der Säulenträger wurde bezüglich seiner Baugröße (thermische Masse) und der Wirksamkeit der Kühllamellen zur Wärmeabfuhr optimiert. In Abb. 3 sind Schema und Bild eines optimierten Versuchsmusters dargestellt. Die GC-Säulen werden hier auf einen mittels 3D-Druck aus Aluminium gefertigten Träger gemeinsam mit einem mineralisolierten Heizdraht gewickelt. Die Temperaturregelung erfolgt auf der Basis des Signals eines Pt-1000. Der Säulenträger ist mit einer integrierten Kühlkörperstruktur ausgestattet und wird durch einen Axialventilator gekühlt. Die Säulen werden thermisch gegen die Umgebung isoliert.
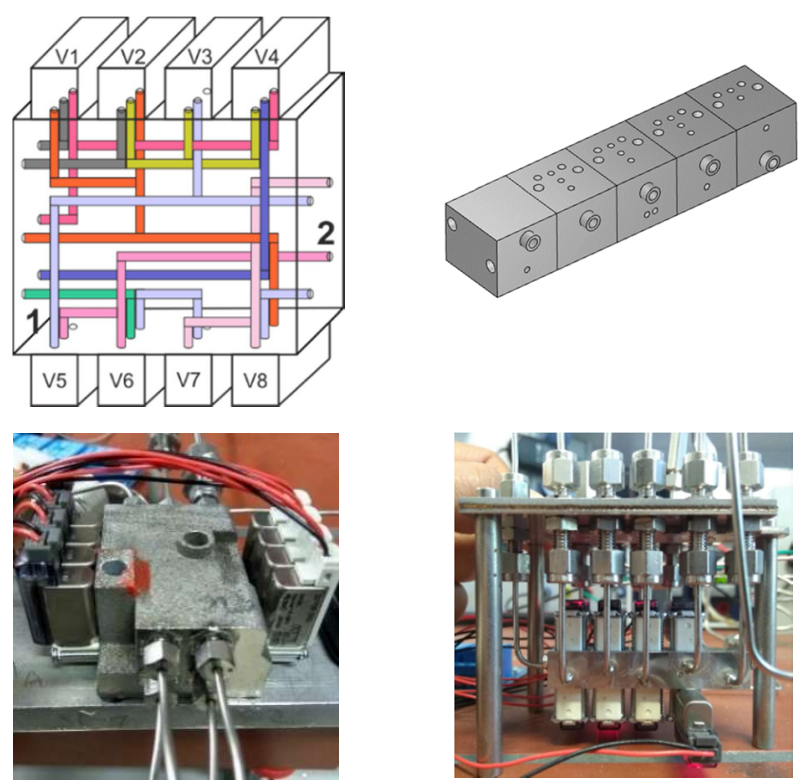

Abb. 2: Schema (oben) und Prototyp (unten) der neu entwickelten Einspritzeinheiten. Am Beispiel des 3Dgedruckten Mini-Manifolds (links) sind die Anschlüsse 
für Probeschleife, Träger-und Messgas, Detektor (1) sowie für die Trennsäulen (2) dargestellt.

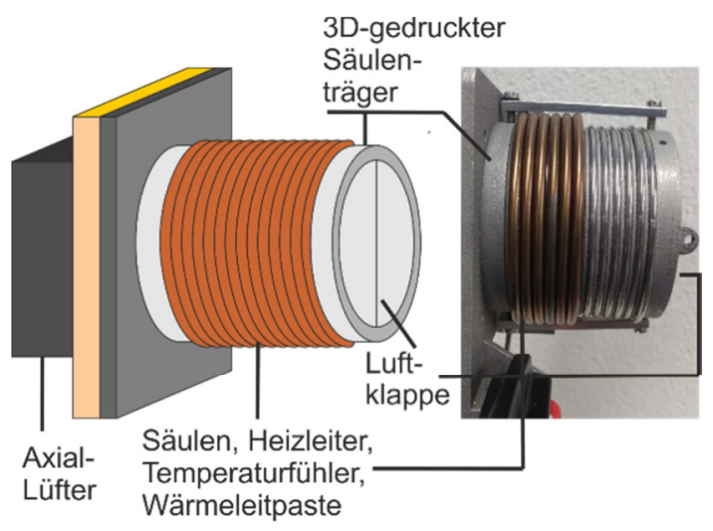

Abb. 3: Schema (oben) und Labor-Versuchsmuster (unten) des miniaturisierten Säulenofens.

\section{Ergebnisse und Diskussion}

\subsection{Einfluss von Detektortemperatur und Polarisati- onsspannung $\left(\mathrm{O}_{2}\right.$-Partialdruck)}

In Abb. 4 sind chromatographische $\mathrm{H}_{2}$ Peaks bei verschiedenen Detektor-Betriebstemperaturen und konstanter Polarisationsspannung sowie gleichbleibender eingespritzter $\mathrm{H}_{2}$-Konzentration $\left(\varphi\left(\mathrm{H}_{2}\right)\right.$ ) dargestellt. Der Trägergas-Volumenstrom betrug $10,5 \mathrm{ml} / \mathrm{min}$. Die Ergebnisse belegen, dass Fläche und Höhe der $\mathrm{H}_{2}$-Peaks bei steigender Detektortemperatur abnehmen. Darüber hinaus wird ein Tailing am Peakende beobachtet, das sich mit steigender Temperatur verringert und deutlich verlängert (siehe auch Detaildiagramm in Abb. 4).

Da das während des Tailings titrierte $\mathrm{H}_{2}$ zur Analytmenge gehört und bei Beendigung der Integration mit Beginn des Sauerstoffpeaks nur unvollständig berücksichtigt wird, werden die Peaks bei verlängertem Tailing kleiner. Daher wird davon ausgegangen, dass eine bestimmte Menge an eingebrachtem $\mathrm{H}_{2}$ (ca. $10 \%$ beim Vergleich der Peaks bei 750 und $600{ }^{\circ} \mathrm{C}$ ) in der Nähe der Messelektrode im Detektor temporär gespeichert wird. Wie in Abb. 5 dargestellt, besteht die Grenzfläche zwischen Pt-Netz-Elektrode und Festelektrolyt aus einer porösen YSZ-Schicht, die auf das YSZ-Rohr gesintert ist und so die für den Sauerstofftransfer wichtige Dreiphasengrenzlinie erhöht. Das gespeicherte $\mathrm{H}_{2}$ eluiert aus dieser Schicht und führt zum Peaktailing. Dieses Phänomen konnte mit den in [12] berichteten Ergebnissen korreliert werden, die aus Untersuchungen mit zyklischer Voltammetrie (CV) an einem baugleichen Detektor bei vergleichbaren Messgas-Volumenströmen resultierten. Diese Ergebnisse belegen eine Zunahme von $\mathrm{H}_{2-}$ bedingten Peakhöhen und -flächen bei steigenden Temperaturen und eine Zwischenspeicherung von $\mathrm{H}_{2}$ an der Elektrode. Bei $600{ }^{\circ} \mathrm{C}$ entspricht die im Peak gefundene Ladungsmenge nahezu der eingespritzten $\mathrm{H}_{2}$-Menge nach GI. 1.

Untersuchungen des Detektorverhaltens bei wechselnden Polarisationsspannungen im Bereich $-300 \mathrm{mV}\left(\mathrm{pO}_{2}=\right.$
$25 \mathrm{mPa})$ bis $-450 \mathrm{mV}\left(\mathrm{pO}_{2}=0,27 \mathrm{mPa}\right)$ belegen, dass das oben beschriebene Peaktailing davon nicht beeinflusst wird. Die Wahl der Polarisationsspannung wirkt sich im untersuchten Bereich nicht auf die $\mathrm{H}_{2}$-Peakparameter aus.

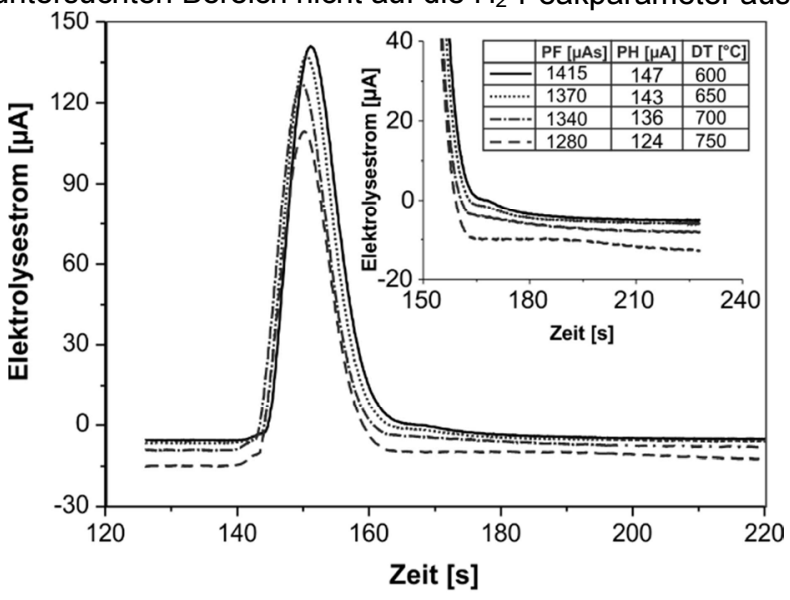

Abb. 4: $\mathrm{H}_{2}$-Peaks in Abhängigkeit von der Detektortemperatur, $\mathrm{PF}=$ Peakfläche, $\mathrm{PH}=$ Peakhöhe, $\mathrm{U}_{\mathrm{P}}=-450 \mathrm{mV}$, $\varphi\left(\mathrm{H}_{2}\right)=180$ Vol.-ppm, Säulenlänge $=2 \mathrm{~m}$.

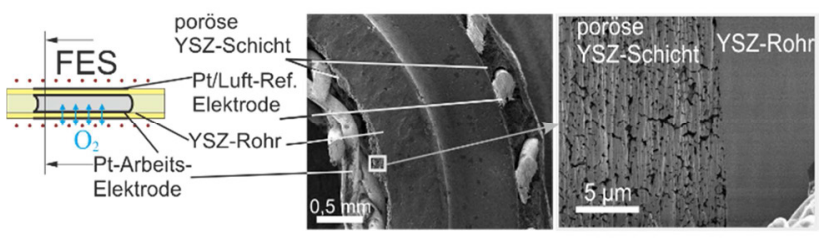

Abb. 5: Schematische Darstellung des Detektors und REM-Aufnahmen der Grenzfläche zwischen Pt-Netzelektrode und YSZ-Rohr mit der porösen aufgesinterten YSZ-Schicht.

\subsection{Einfluss der $\mathrm{H}_{2}$-Konzentration}

Das Ansprechverhalten des FES Detektors wurde bei verschiedenen injizierten Wasserstoffkonzentrationen im Bereich von 0,2-180 Vol.-ppm bei gleichbleibenden übrigen Parametern charakterisiert. Die entsprechenden Chromatogramm-Ausschnitte mit mittleren Peakflächen und Peakhöhen sind in Abb. 6a dargestellt. Die Ergebnisse zeigen, dass das in Abschnitt 3.1 beschriebene Peaktailing auch bei fast allen niedrigeren Konzentrationen auftritt. Darüber hinaus treten bei Konzentrationen zwischen 20 und 180 Vol.-ppm, Front- und Backend-Stufen im Peak auf, die wahrscheinlich auf die spezifische Säulendurchströmung zurückzuführen sind und bei $\mathrm{H}_{2}$ Spurenkonzentrationen $<10$ Vol.-ppm verschwinden.

Der in Abb. 6b für $700{ }^{\circ} \mathrm{C}$ aufgeführte Vergleich der ausgewerteten Peakflächen mit den injizierten $\mathrm{H}_{2}$ Konzentrationen gemäß GI. 1 belegt eine nahezu vollständige coulometrische Umsetzung des Analytes. Diese vollständige Oxidation bewirkt einerseits eine maximale Genauigkeit des Detektors. Andererseits ist damit ein weitgehend kali-brierfreier Betrieb des gesamten Messsystems möglich. Die geringsten Abweichungen zwischen gemessenen und berechneten Ladungsmengen von $<4$ $\%$ wurden bei Spurenkonzentrationen $<10$ Vol.-ppm und niedrigen Temperaturen $\left(\sim 60{ }^{\circ} \mathrm{C}\right)$ gefunden. Die erhöh- 
ten Schwankungen in dem Basislinienstrom (und dadurch verschlechtertes Signal-Rausch Verhältnis) und erhöhte Speichereffekte mit steigenden Temperaturen erzeugen den erhöhten Abweichungen bei Spurenkonzentrationen.
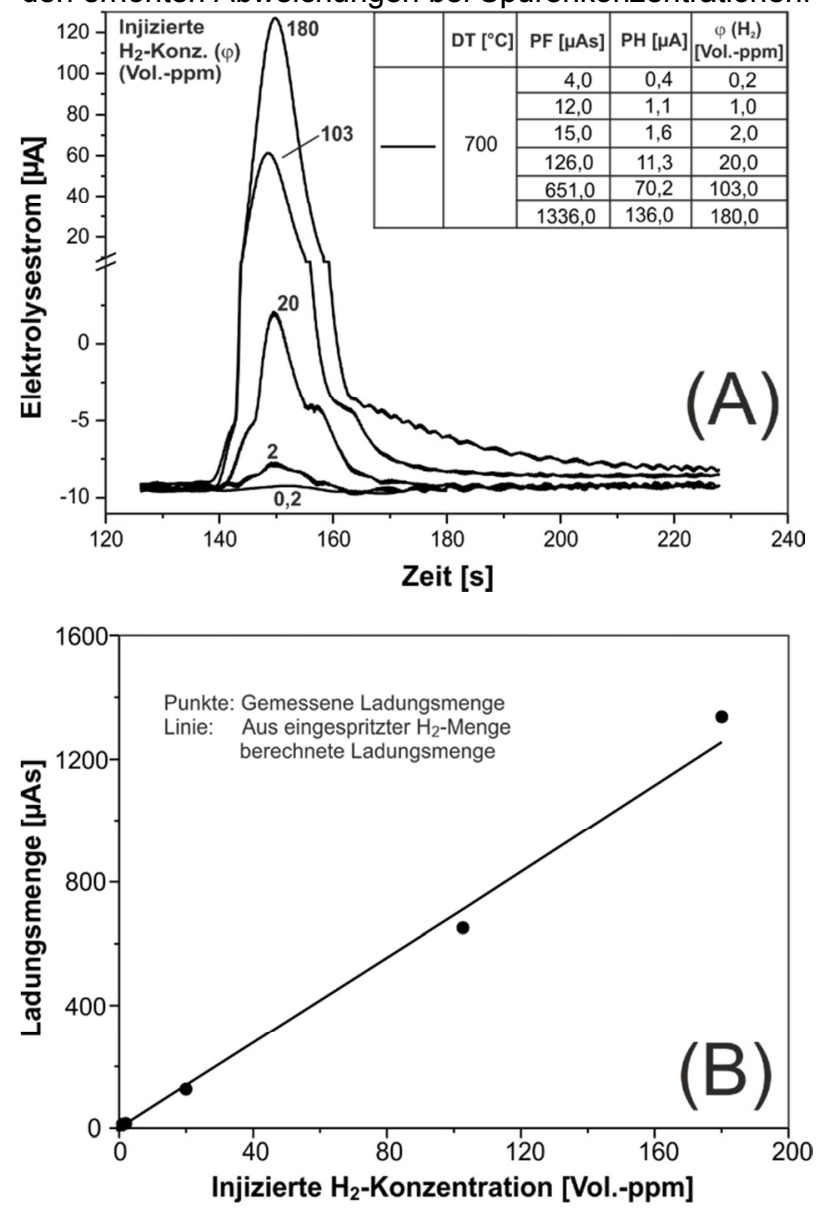

Abb. 6 (A): Chromatographische $\mathrm{H}_{2}$-Peaks mit Peakflächen $(\mathrm{PA})$ und Peakhöhen $(\mathrm{PH})$ bei variierender injizierter Konzentration $\varphi\left(\mathrm{H}_{2}\right)=0,2-180$ Vol.-ppm, $\mathrm{U}_{\mathrm{P}}=-450$ $\mathrm{mV}$, Detektortemperatur $(\mathrm{DT})=700^{\circ} \mathrm{C}$. (B) Vergleich zwischen Ladungsmengen aus dem gemessenen Peakflächen und gemäß Faraday'schem Gesetz berechneten Ladungsmengen bei o.g. Bedingungen.

\subsection{Einfluss von Trägergas-Volumenstrom und Trennsäulenlänge}

Die Untersuchung der Abhängigkeiten der Peakparameter vom Trägergasvolumenstrom bei Verwendung gepackter Trennsäulen der Länge $2 \mathrm{~m}$ ergab nur sehr geringfügige Einflüsse dieses Parameters wie in [11] beschrieben. Es konnte gezeigt werden, dass bei den getesteten Trägergas-Volumenströmen im Bereich 6-16 ml/min der Analyt immer vollständig elektrochemisch titriert wird. Darüber hinaus führen höhere Trägergas-Volumenströme immer zu einem vergrößerten Signal-Rausch-Verhältnis (SNR, gemäß ISO 11843-1). Weiterhin wurde gefunden, dass sich das SNR auch mit sinkender Detektortemperatur vergrößert, da Basisstromschwankungen und Rauschamplituden hauptsächlich von diesem Parameter abhängen [10]. Aus den Ergebnissen konnten wesentliche Schlussfolgerungen für die Optimierung der Konstruktion eines miniaturisierten Messsystems abgeleitet werden, um ein angemessenes SNR bei vollständigem $\mathrm{H}_{2}$-Umsatz, kurzer Analysezeit und niedriger Nachweisgrenze im ppbBereich zu gewährleisten.

Zur Miniaturisierung des Säulenofens wurden Detektorsignale und Trennleistung verkürzter GC-Säulen der Länge $1 \mathrm{~m}$ unter variierenden Messbedingungen charakterisiert. Für diese Säulenkonfiguration sind $\mathrm{H}_{2}$-Peaks bei 3 verschiedenen Trägergas-Volumenströmen (16,5, 25,5 und $31,5 \mathrm{ml} / \mathrm{min}$ ) und 3 verschiedenen injizierten $\mathrm{H}_{2-}$ Konzentrationen in Abb. 7 aufgeführt. Wie erwartet, führt diese Halbierung der Säulenlänge zur Verringerung der Retentionszeit aller Peaks um etwa den Faktor 4.

Die in Abb. 7 aufgeführten Werte für die berechneten Peakflächen führen zu der Vermutung, dass in diesen verkürzten Säulen verschiedene Prozesse zu einer deutlicheren Beeinflussung der Menge titrierten $\mathrm{H}_{2}$ durch den Trägergasvolumenstrom führen. Bei Konzentrationen im Bereich um 20 Vol.-ppm führen das verlängerte Tailing bei niedrigen Volumenströmen und die Überschneidung der $\mathrm{H}_{2-}$ und $\mathrm{O}_{2}$ Peaks bei höheren Volumenströmen zu einer Abnahme der Peakfläche. Im Spurenkonzentrationsbereich sind die Speichereffekte weniger ausgeprägt. Daher weisen die Peaks für 0,2 Vol.-ppm bei hohen Volumenströmen und geringerer Säulenlänge eine größere Höhe und ein günstigeres SNR auf als bei längeren Säulen.

In Abb. 8 sind $\mathrm{H}_{2}$-Peaks beim höchsten getesteten Trägergas-Volumenstrom und drei verschiedenen Detektortemperaturen aufgeführt. Um eine konstante Basislinie im Peakbereich zu gewährleisten, wurde die Elektrodenpolarisation am FES etwa 1 min vor der Probeinjektion gestartet, was beim Einsatz von Säulen der Länge $2 \mathrm{~m}$ nicht notwendig war.

Es konnte gezeigt werden, dass die geringste Differenz zwischen titrierter und injizierter $\mathrm{H}_{2}$-Menge bei der $\mathrm{H}_{2}$ Konzentration 0,2 Vol.-ppm und der Detektortemperatur $600{ }^{\circ} \mathrm{C}$ beim höchsten Volumenstrom reproduzierbar entsteht. Dieses Ergebnis zeigt, dass die Zielstellung der Geräteentwicklung im Hinblick auf Miniaturisierung und erhöhte Sensitivität mit kürzeren Säulen eher erreicht werden kann.

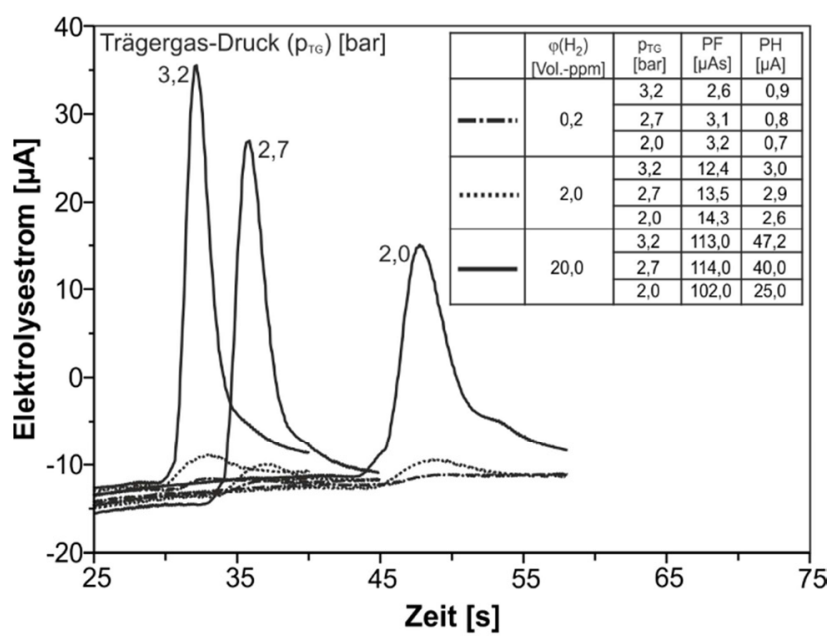


Abb. 7: $\mathrm{H}_{2}$-Peaks mit entsprechenden Parametern bei verschiedenen Trägergas-Einlassdrücken, Säulenlänge $=1 \mathrm{~m}$, $\mathrm{U}_{\mathrm{P}}=-450 \mathrm{mV}$, Detektortemperatur $=650{ }^{\circ} \mathrm{C}$.
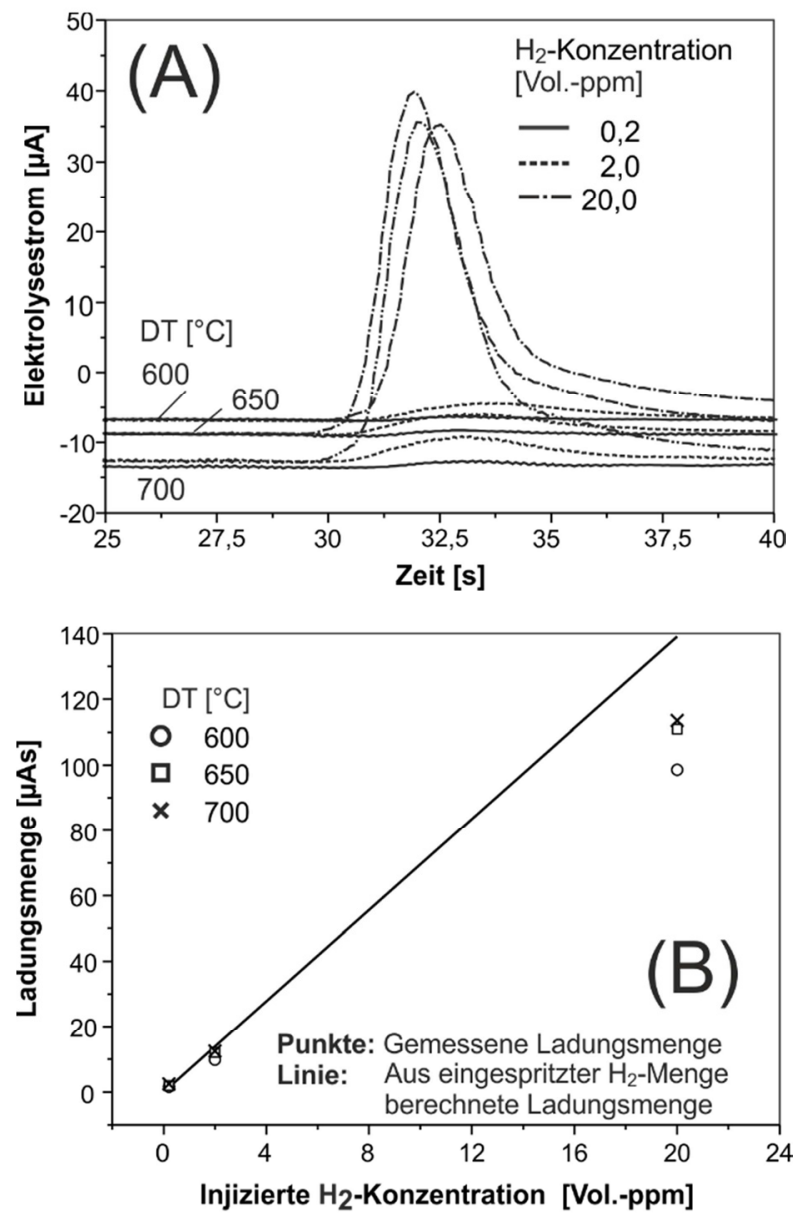

Abb. 8 (A): $\mathrm{H}_{2}$-Peaks bei verschiedenen Detektortemperaturen (DT). (B) Peakflächen und Peakhöhen bei variierenden Temperaturen und Vergleich mit den injizierten $\varphi\left(\mathrm{H}_{2}\right)$. Trägergas-Einlassdruck $=3,2 \mathrm{bar}$, Säulenlänge $=1 \mathrm{~m}, \mathrm{U}_{\mathrm{P}}=-450 \mathrm{mV}$.

\subsection{Charakterisierung der miniaturisierten Injekti- onseinheit}

Das neuentwickelte Mikro-Manifold wurde wie in [11] beschrieben bezüglich Gasdichtigkeit und Gasausbreitung getestet. Die mittels Heliumlecktest ermittelten Leckageraten zwischen den inneren Kanälen und an den äußeren Anschlüssen betragen etwa $10^{-8} \mathrm{mBar} \cdot \mathrm{L} / \mathrm{s}$. Der an der komplett montierten Einspritzeinheit gemessene Sauerstoffeintrag ist kleiner als 0,1 Vol.-ppm und somit vernachlässigbar. Um den Injektionsverlauf zu charakterisieren, wurden potentiostatische Scans über mehrere Injektionszyklen durchgeführt. In Abb. 9 sind entsprechende $\mathrm{H}_{2}$ Peaks für zwei Trägergas-Volumenströme (10 und 30 $\mathrm{ml} / \mathrm{min}$ ) dargestellt. Die Ergebnisse zeigen eine stabile Titrationsstrom-Basislinie sowie eine zu erwartende Peakhöhenänderung mit zunehmendem Gas-Volumenstrom. Weiterhin treten negative Strompeaks während der ersten Einspritzzyklen nach Start des Trägergasstroms auf. Diese werden durch Restsauerstoff in Totvolumina im Ma- nifold und in den Ventilen erzeugt und gehen nach etwa 510 Zyklen vollständig zurück. Bei gleichen Betriebsbedingungen weisen die am Mikromanifold gemessenen $\mathrm{H}_{2}$ Peaks ein deutlich reduziertes Tailing gegenüber den am Minimanifold gefundenen auf.

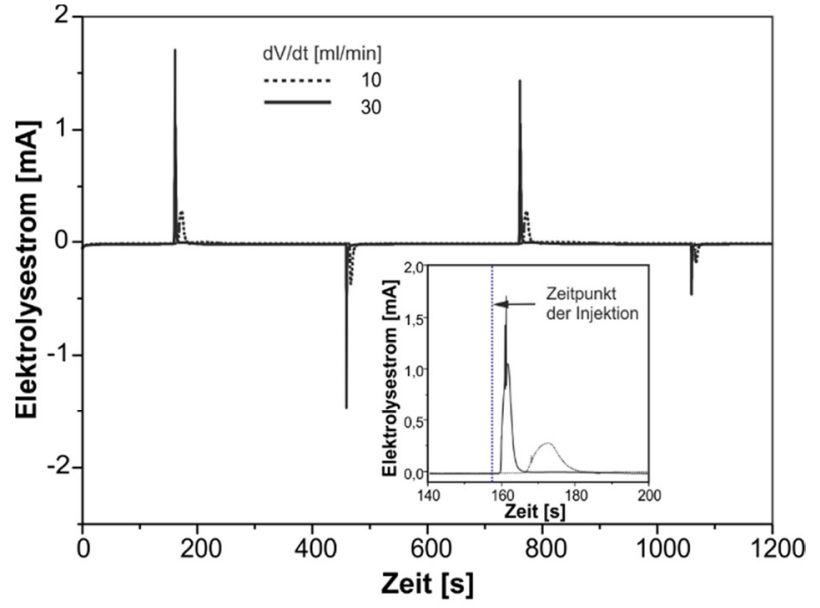

Abb. 9: $\mathrm{H}_{2}$-Peaks nach Einspritzung mittels MikroManifold gemäß Abb. 2 in verschiedene TrägergasVolumenströme $(\mathrm{dV} / \mathrm{dt}), \mathrm{U}_{\mathrm{P}}=-450 \mathrm{mV}$, injiziertes Probevolumen $=1 \mathrm{~mL}, \varphi\left(\mathrm{H}_{2}\right)=300$ Vol.-ppm, Detektortemperatur $=600{ }^{\circ} \mathrm{C}$. Insert: Erster Injektionsschritt vergröBert.

Außerdem deutet die schnellere Verringerung der negativen $\mathrm{O}_{2}$-Peaks beim Mikromanifold auf eine erfolgreiche Verkleinerung der Totvolumina hin. Ebenso verringert sich die Retentionszeit bei Einsatz des feinwerktechnisch hergestellten Mikromanifolds. Ein wesentliches Problem bei der Inbetriebnahme des 3D-gedruckten Minimanifolds ergab sich mit der mangelnden Dichtigkeit zwischen den inneren Kanälen, da Gas durch technologiebedingte Poren im Sinterkörper diffundieren konnte. Dieser Nachteil konnte zwar durch eine nachträgliche Auskleidung der Kanäle mit Epoxidharz behoben werden. Diese relativ aufwendige Methodik ist jedoch für eine Weiterentwicklung ungeeignet. Das feinwerktechnisch aus Vollmaterial hergestellte Mikromanifold weist diesen Nachteil nicht auf und wurde wegen seiner Vorteile für die weitere Geräteentwicklung favorisiert.

\subsection{Charakterisierung des miniaturisierten Säulen- ofens}

Die in [11] beschriebene Grundkonstruktion des miniaturisierten Säulenofens konnte in ersten Labortests erfolgreich im Hinblick auf die angestrebten Aufheiz- und Abkühlraten charakterisiert werden. Das nun vorliegende zweite Versuchsmuster konnte wegen der getesteten Trennleistung der kürzeren GC-Säulen deutlich miniaturisiert werden, so dass dessen Abmessungen 112 × 80 × 88 $\mathrm{mm}^{3}$ betragen und die thermische Masse des Säulenträgers um ca. $65 \%$ geringer ausfällt. Damit reicht die auf 90 W begrenzte Heizleistung für den Betrieb aus. Der 3Dgedruckte Aluminium-Säulenträger weist wegen seiner 
rauen Oberflächenbeschaffenheit eine deutlich verbesserte Wärmeabfuhr auf.

\section{Zusammenfassung}

Das Messverhalten eines coulometrischen Festelektrolyt-Detektors (FES) in Kombination mit einer chromatographischen Trennung des Messgases wurde unter Variation von Detektortemperatur, Polarisationsspannung, eingespritzter $\mathrm{H}_{2}$-Konzentration, Trägergas-Volumenstrom und Trenn-säulenlänge untersucht. Diese Charakterisierung ermöglichte die Aufklärung von Zusammenhängen zwischen verschiedenen diffusions- und reaktionskinetischen Prozessen an den Messelektroden und den chromatographischen Trennprozessen. Die Resultate wurden für eine materialtechnische und konstruktionsbezogene Optimierung des Messsystems im Hinblick auf eine höhere Selektivität und Langzeitstabilität sowie eine niedrigere untere Nachweisgrenze genutzt. Bei Detektortemperaturen über $700{ }^{\circ} \mathrm{C}$ wurde ein ausgeprägtes $\mathrm{H}_{2}$-Peak-Tailing beobachtet, was auf eine Zwischenspeicherung und eine anschließende langsame Freisetzung von $\mathrm{H}_{2}$ aus einem elektrodennahen Speicher hinweist. Es wird davon ausgegangen, dass diese Speicherung in einer porösen Keramikschicht zwischen der Elektrode und dem Elektrolyten erfolgt. Dieses Ergebnis deckt sich mit den dynamischen Messungen mittels zyklischer Voltammetrie an einem ähnlichen Detektor. Dieses Tailing kann nahezu vollständig vermieden werden, indem das Volumen der porösen Schicht in dem miniaturisierten FES verringert wird und der Detektor bei $<=650{ }^{\circ} \mathrm{C}$ betrieben wird. Die bei verschiedenen $\mathrm{H}_{2}$-Konzentrationen und der Detektortemperatur $600{ }^{\circ} \mathrm{C}$ gemessenen Peakflächen zeigten bei einer zufriedenstellenden Übereinstimmung mit dem Faraday'schen Gesetz. Diese dadurch gegebene hohe Detektorstabilität ermöglicht dessen kalibrierfreien Betrieb über lange Laufzeiten. Die Standardabweichung der an Trennsäulen der Länge $2 \mathrm{~m}$ gefundenen Retentionszeit der Wasserstoffpeaks beträgt über 400 Messungen etwa $1,5 \mathrm{~s}$.

Für die Miniaturisierung des Säulenofens wurden die Trennleistung sowie die zugehörigen Betriebsparameter (Eingangsdrücke und Volumenströme) von Trennsäulen der Länge $1 \mathrm{~m}$ getestet. Wie erwartet, reduzieren sich die Retentionszeiten für den $\mathrm{H}_{2}$-Peak um bis zu den Faktor 4 und die Trennleistung geht bei Konzentrationen $>10$ Vol.ppm zurück. Gleichzeitig erhöht sich die Messgenauigkeit für Spurenkonzentrationen ( $<2$ Vol.-ppm), insbesondere bei höheren Trägergasvolumenströmen (30 ml/min) und niedrigen Detektortemperaturen $\left(600^{\circ} \mathrm{C}\right)$.

Zwei hochminiaturisierte Probeninjektionssysteme wurden mittels 3D-Druck bzw. mit Präzisionsbohrtechnik hergestellt und im Hinblick auf ihre Dichtigkeit, Totvolumina und Gasdispersion untersucht. Der mittels Feinwerktechnik in Modulbauweise gefertigte Prototyp zeichnet sich dabei durch geringe Totvolumina, niedrige Leckageraten und geringe Peakverbreiterung aus. Ein miniaturisierter Säulenofen wurde mit Hilfe eines 3D-gedruckten Alumini- umträgers für die Trennsäulen hergestellt und erfolgreich im Labor erprobt.

\section{Danksagung}

Die präsentierte Arbeit wurde im Rahmen eines vom Bundesministerium für Bildung und Forschung geförderten Vorhabens unter dem Förderkennzeichen 03ZZ0724A durchgeführt. Für die materialbezogenen Untersuchungen und Analysen von Herrn Frank Altmann und seinem Team am Fraunhofer-Institut für Mikrostruktur von Werkstoffen und Systemen IMWS in Halle sind die Autoren sehr dankbar.

Darüber hinaus danken die Autoren der CS Chromatographie Service $\mathrm{GmbH}$, Langerwehe, und der ZiroxSensoren \& Elektronik $\mathrm{GmbH}$, Greifswald, für die professionelle gerätebezogene Unterstützung.

\section{Literatur}

[1] EDWARDS, P.P; KUZNETSOV, V.L.; DAVID W.I.F.: Hydrogen Energy. In: Phil. Trans. R. Soc. A (2007), Nr. 365, S. 1043-1056

[2] Ramachandran R.; Menon R.K.: An overview of industrial uses of Hydrogen. In: International Journal of Hydrogen Energy (1998), Nr. 23, S. 593-598

[3] DAGDOUGUI H.: Models, methods and approaches for the planning and design of the future hydrogen supply chain In: International Journal of Hydrogen Energy (2012), Nr. 37, S. 5318-5327

[4] SINIGAGLIA T.; LEWISKI F.; EDUARDO M.; MARTINS S.; CEZAR J.; SILUK M.: Production, storage, fuel stations of hydrogen and its utilization in automotive applications- a review. In: International Journal of Hydrogen Energy (2017) 42, Nr. 39, S. 24597-24611

[5] MAROUFMASHAT A.; FOWLER M.: Transition of Future Energy System Infrastructure through Power-toGas Pathways. In: Energies (2017), Nr. 10(8), 10891111

[6] PASMAN H. J.: Challenges to improve confidence level of risk assessment of hydrogen technologies. In: International Journal of Hydrogen Energy (2011), Nr. 36, S. 2407-2413

[7] HÜBERT T.; BOON-BRETT L.; PALMISANO V.; BADER M.A.: Developments in gas sensor technology for hydrogen safety. In: of Hydrogen Energy (2014), Nr. 39 (35), S. 20474-20483

[8] BOON-BRETT L.; BOUSEK J.; BLACK G.; MORETTO P.; CASTELLO P.; HÜBER T.; BANACH U.: Identifyingperformance gaps in hydrogen safety sensor technology for automotive and stationary applications. In: International Journal of Hydrogen Energy (2010), Nr. 35 (1), S. 373-384

[9] TESKE K.; POPP P.; BAUMBACH J.: Solidstatecoulometric cell as detector for gas chromatography. In: Journal of Chromagraphy (1986), Nr. 360, S. 417-420

[10] SCHELTER M.; ZOSEL J.; OELSSNER W.; GUTH U.; MERTIG M.: A solid electrolyte sensor for trace gas analysis. In: Sensors and Actuators B: Chemical (2013), Nr.187, S. 209-214

[11] SOOD P.; ZOSEL J.; MERTIG M.; OELSSNER W.; KLOCKOW A.; HERRMANN O.; WORATZ M.: Devel- 
opment and characterization of a highly selective hydrogen sensor system electrochemical methods. In: 20. GMA/ITG-Fachtagung Sensoren und Messsysteme (2019), Nr. 3.3.4, S. 264-270

[12] RUCHETS A.; DONKER N.; SCHÖNAUER-KAMIN D.; MOOS R.; ZOSEL J.; GUTH U.; MERTIG M.: Selectivity improvement towards hydrogen and oxygen of solid electrolyte sensors by dynamic electrochemical methods. In: Sensors and Actuators B: Chemical (2019), Nr. 290, S. 53-58 